\title{
CORONARY ARTERY BYPASS GRAFTING WITHOUT CARDIOPULMONARY BYPASS IN PHEOCHROMOCYTOMA
}

\author{
Amit Roy, MA, MRCS, Avinash Sinha, FRCA, Miles Scholar, BSc, MB BS, Ken Macleod, FRCA, and
} Brian Glenville, MS, FRCS, London, United Kingdom

Coronary artery disease requiring surgery combined with a pheochromocytoma is a rare scenario, which presents difficulties in controlling life-threatening paroxysmal alterations in blood pressure and heart rate. All reported cases of coronary artery bypass grafting (CABG) before removal of a pheochromocytoma have involved the institution of cardiopulmonary bypass (CPB). Severe hypertension and tachyarrhythmias have been reported after cessation of $\mathrm{CPB}$ in reported cases of $\mathrm{CABG}^{1,2}$ and mitral valve replacement. ${ }^{3}$ To our knowledge, however, this is the first reported case of $\mathrm{CABG}$ in the presence of a pheochromocytoma without the use of CPB.

Clinical summary. A 65-year-old man with a 2-year history of angina (New York Heart Association class III) was admitted for repeat angiography after two previous angioplasties. Medical history included polycythemia rubra vera, hypertension treated with $\beta$-blockers, and profuse sweating. Preceding the angiogram, he was noted to have labile blood pressure and postural hypotension. Subsequent cardiac catheterization provoked extreme hypertensive and hypotensive paroxysms. On further investigation, plasma and urinary catecholamine levels were found to be significantly elevated with a plasma epinephrine concentration of $208 \mathrm{pg} / \mathrm{mL}$ and a norepinephrine concentration of $12,573 \mathrm{pg} / \mathrm{mL}$ (normal ranges: $20-100 \mathrm{pg} / \mathrm{mL}$ and $200-500 \mathrm{pg} / \mathrm{mL}$, respectively). Subsequent radio-labeled metaiodobenzylguanidine scintigra-

From the Cardiothoracic Unit, St Mary's Hospital, London, United Kingdom.

Received for publication May 11, 2000; accepted for publication May 17, 2000.

Address for reprints: Amit Roy, MA, MRCS, 6 Heritage Ct, Trundleys Rd, Surrey Quays, London, SE8 5JX, United Kingdom (E-mail: amit@amitroy.freeserve.co.uk).

J Thorac Cardiovasc Surg 2000;120;826-8

Copyright () 2000 by The American Association for Thoracic Surgery

$0022-5223 / 2000 \$ 12.00+0 \quad \mathbf{1 2 / 5 4 / 1 0 8 6 9 7}$

doi: $10.1067 / \mathrm{mtc} .2000 .108697$ phy, venous plasma sampling, and pelvic gadoliniumenhanced magnetic resonance imaging revealed a 4-cm extraadrenal actively secreting mass consistent with a pheochromocytoma, situated just above the level of the aortic bifurcation between the aorta and inferior vena cava (Fig 1).

Echocardiography revealed a minimally dilated left ventricle with an ejection fraction of $60 \%$. Angiography demonstrated proximal stenoses of greater than $80 \%$ in the left anterior descending artery, its first diagonal branch, and the first obtuse marginal artery. In view of the significant coronary artery disease and unstable functioning pheochromocytoma, we decided to perform off-pump CABG before removing the pheochromocytoma and thus avoid CPB.

Preoperative preparation included administering oral phenoxybenzamine $10 \mathrm{mg} 3$ times a day for 2 weeks before the operation, along with atenolol $25 \mathrm{mg}$ once a day once adequate $\alpha$-blockade had been achieved. Before induction of anesthesia, infusions of phentolamine, sodium nitroprusside, norepinephrine, epinephrine, and lidocaine were prepared so that they could be administered immediately if needed to correct any acute changes in blood pressure or tachyarrhythmia. Anesthesia was induced with etomidate and alfentanil and maintained with $50 \%$ nitrous oxide and $0.5 \%$ to $1.5 \%$ enflurane in oxygen. Vecuronium was used for muscle relaxation. High lumbar epidural analgesia was administered with a 100$\mathrm{mg}$ bupivacaine loading dose, followed by an infusion of $0.166 \%$ bupivacaine with $1 \mathrm{mg}$ of diamorphine per $6 \mathrm{~mL}$ of normal saline solution, infused at a rate of 0 to $6 \mathrm{~mL} / \mathrm{h}$. In addition to providing intraoperative and postoperative analgesia, the epidural would also produce a sympathetic blockade, which would obtund any swings in blood pressure associated with surges in catecholamine release from the tumor. The use of anticholinergic agents, halothane, sympathomimetic neuromuscular blockade, and morphine was avoided.

Changes in blood pressure and pulse throughout the procedure were recorded (Fig 2). After routine sternotomy and internal thoracic artery harvesting, CABG was performed, without instituting $\mathrm{CPB}$, by means of a compression stabiliz- 


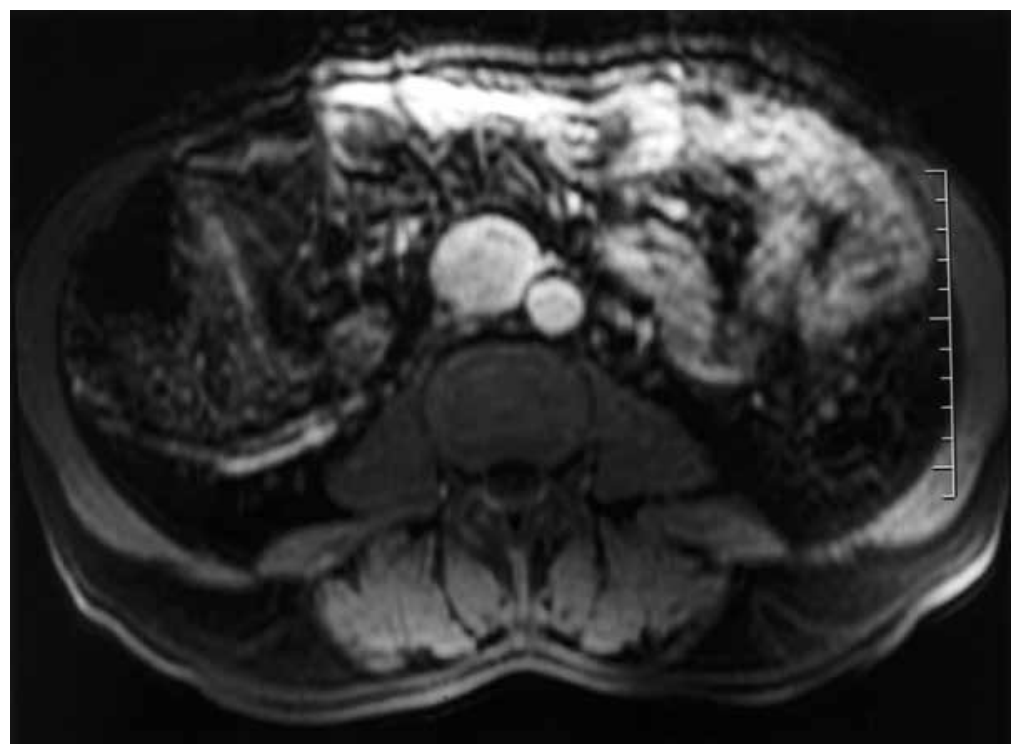

Fig 1. Gadolinium-enhanced pelvic magnetic resonance imaging scan. A 4-cm enhanced round mass can be seen to the left of the abdominal aorta, situated just above the level of the aortic bifurcation. This mass is consistent with an actively secreting pheochromocytoma.

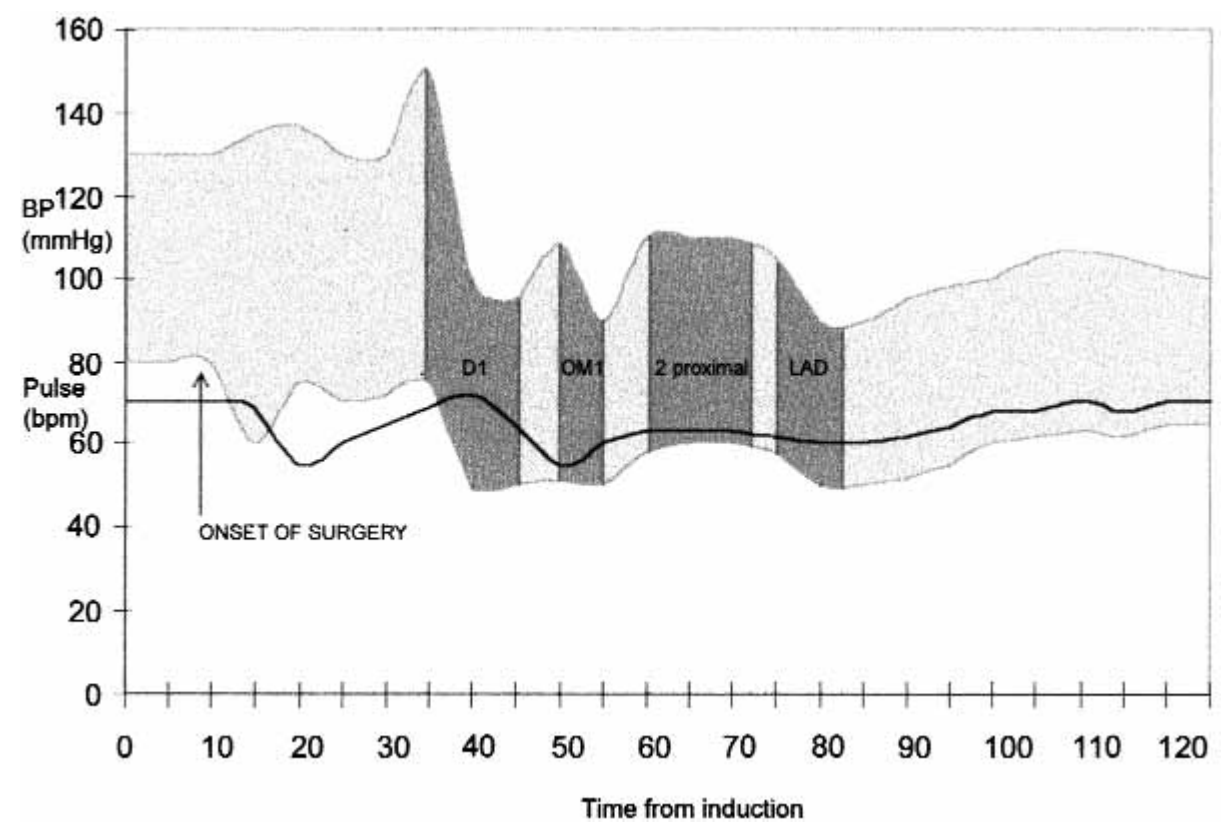

Fig 2. Graph showing intraoperative changes in systolic and diastolic blood pressure (light gray areas) and pulse rate (bold line) with time. Dark gray areas represent timing of graft anastomoses: D1, First diagonal branch of left anterior descending artery; $O M 1$, first obtuse marginal branch of circumflex artery; 2 proximal; two aorta-saphenous proximal anastomoses; $L A D$, left anterior descending artery; $B P$, blood pressure.

ing device (Access Ultima, CardioThoracic Systems, Inc, Cupertino, Calif) to construct all coronary anastomoses. The first graft to be carried out was a long saphenous vein graft to the first diagonal branch. On application of the stabilizing device, blood pressure fell slightly. This fall was no more than was expected from our previous experience in routine offpump CABG operations. Once the stabilizing device was released after completion of the anastomosis, blood pressure rose to normal levels. This pattern was repeated again as expected during the construction of the graft to the first 
obtuse marginal branch, also with saphenous vein. To facilitate grafting the circumflex territory, we opened the right pleura wide to allow partial dislocation of the heart into the right hemithorax to prevent significant obstruction to venous return. Next, both aorta-saphenous vein anastomoses were constructed with a side-biting clamp on the aorta, during which time the blood pressure remained normal. Finally, the left internal thoracic artery was grafted to the left anterior descending artery with minor alteration in blood pressure, which again resolved on removal of the stabilizing device. No intraoperative inotropic drugs or blood pressure-lowering agents were necessary during the procedure.

The patient was transferred to the intensive care unit awake and was extubated immediately. Other than the development of atrial fibrillation, which was treated with amiodarone, the remainder of the postoperative course was uneventful. The patient was discharged on day 7 and was readmitted 1 month later for removal of the pheochromocytoma, which was uneventful.

Discussion. Pheochromocytoma is a rare condition accounting for $0.1 \%$ of cases of hypertension. In any surgical procedure on such a patient, the risks of cardiovascular collapse, myocardial infarction, and cerebrovascular accident are significantly elevated. On occasion, a patient requiring removal of a pheochromocytoma also needs surgical coronary revascularization. The discontinuation of CPB in such cases has been associated with precipitation of severe hypertension and tachycardia. The basis for this reaction is not clear. It is interesting that anesthetic induction with fentanyl was used in two reported cases. ${ }^{1,3}$ It has been suggested that although fentanyl attenuates catecholamine release before $\mathrm{CPB}$, large increases in circulating catecholamines do occur during $\mathrm{CPB} .{ }^{4}$ Hypothermia, used routinely during $\mathrm{CPB}$, has also been reported to provoke catecholamine secretion by pheochromocytoma. ${ }^{5}$ By performing off-pump CABG, we avoided the hazardous effects of CPB on pheochromocytoma-induced changes in systemic vascular resistance and hence blood pressure. The use of the off-pump technique resulted in only minimal changes in blood pressure, which occurred during cardiac manipulation and stabilizing device application.

Hence, with good preoperative preparation and appropriate anesthesia, off-pump CABG can be done safely in a patient with pheochromocytoma, avoiding $\mathrm{CPB}$. This operation can be done before proceeding to surgical removal of the pheochromocytoma, thus reducing the cardiac risks of the pheochromocytoma operation.

\section{REFERENCES}

1. Brown P, Caplan RA. Recognition of an unsuspected phaeochromocytoma during elective coronary artery bypass surgery. Can Anaesth Soc J 1986;33:785-9.

2. Dunn EJ, Wolff RK, Wright CB, et al. Presentation of undiagnosed phaeochromocytoma during coronary artery bypass surgery. J Cardiovasc Surg 1989;30:284-7.

3. Fenje N, Lee LW, Jamieson WRE, et al Phaeochromocytoma and mitral valve replacement. Can J Anaesth 1989;36:198-9.

4. Stanly TH, Philbin DM, Coggins CH. Fentanyl-oxygen anaesthesia for coronary artery surgery: cardiovascular and ADH responses. Can Anaesth Soc J 1979;26:168-72.

5. DeCourcy JL, DeCourcy CB. Pheochromocytoma and the general practitioner. Cincinnati: Barclay Newman; 1952. p.32. 